\title{
Consumo de drogas por vía intravenosa y sida en Edimburgo: maximizar los beneficios y minimizar los riesgos*
}

\author{
LEWIS, ROGER \\ Centre for HIVIAIDS and Drug Studies. Edimburgo. \\ Enviar correspondencia a: \\ Roger Lewis. Observatorio Europeo para la Droga y las Drogadicciones. Rua Cruz de Sta. Apolinia, 23. 1149 Lisboa
}

\begin{abstract}
Resumen:
Desde 1983, en algunas regiones de Escocia, a diferencia de Inglaterra, se ha producido una mayor expansión del SIDA debido al consumo de drogas. En la actualidad, el número de enfermos de SIDA, en Edimburgo y Lothian, asciende a 1.105 personas. A partir de 1985 se han ido incrementado las medidas dirigidas a paliar este problema, entre las que cabe mencionar el intercambio de jeringuillas usadas por otras nuevas, la prescripción de metadona, la creación de instituciones comunitarias antidroga y las campañas de prevención. Desde 1988, el número de afectados por el HIV, en relación con el consumo de drogas, ha disminuido notablemente. No obstante, la transmisión por contacto sexual sigue siendo motivo de preocupación, especialmente entre las parejas de los drogadictos seropositivos que se inyectan por vía intravenosa.
\end{abstract}

Palabras clave: drogas, intravenosa, SIDA, reducción de los daños, tratamiento, sexo.

\section{Summary:}

In some regions of Scotland, unlike England, the AIDS epidemic related to the use of drugs is widespread since 1983. At present, Edinburgh and the Lothian region have a population of 1.105 individuals affected by AIDS. A series of preventive measures, including the exchange of used syringes by new ones, the prescription of methadone, community anti-drug organisations, and prevention campaigns have been progressively increased since 1985. The number of individuals affected by HIV due to drug consumption has notably decreased since 1988. Sexual transmission remains, however, an issue of concern, particularly among those who have HIV-positive partners who use injectable drugs.

Key words: heroin addicts, risk reduction, AIDS, syringes exchange, sex, treatment.

tada contra el SIDA, basada en el concepto de reducción de los daños, ha sido determinante para que en los últimos diez años los servicios de asistencia y prevención hayan podido intervenir eficazmente en las prácticas del consumo de drogas. La drogadicción en las cárceles sigue siendo motivo de alarma. Al igual que en otros, dichos servicios han tenido menos éxito al intentar modificar el comportamiento sexual de los toxicómanos por intravenosa.

\section{CONTEXTO}

La población de Edimburgo es de 415.000 habitantes. La región de Lothian a la que pertenece tiene

* Artículo aparecido en Personalità/dipendenze, Vol.4, fasc.ll (1998) 
750.000 habitantes. La población de toda Escocia asciende a 5,4 millones de habitantes. En Escocia, los problemas que ocasiona el abuso de drogas, sobre todo en relación con la epidemia del SIDA, deben considerarse en el contexto de los últimos quince años. Al principio no se tenían noticias sobre el consumo de drogas ilegales en las zonas urbanas desfavorecidas. Sin embargo, a principios de los años 80 surgió un mercado negro de heroína, junto con un rápido aumento del consumo de otras drogas, en particular en Edimburgo y Glasgow. No pasó mucho tiempo antes de que las sustancias opiáceas ilegales y sus consumidores pasaran a formar "parte del paisaje" (Barnard y McKeganey, 1990). Como es habitual, los toxicómanos eran jóvenes desempleados que se inyectaban la droga y que procedían de las zonas urbanas más pobres. En 1986, cuando el paro alcanzó en Edimburgo el $13 \%$, la tasa de parados varones, en una zona con una población de drogadictos y enfermos de SIDA muy elevada, se estimaba en cerca del 32,3\% (Robertson y Bucknall, 1986).

\section{MODALIDADES DEL CONSUMO DE DROGA}

A principios de los 80 , los pequeños traficantes ("camellos") de heroína que la vendían por onzas en Glasgow, la ciudad más grande de Escocia, eran también fuertes consumidores. Otros camellos-consumidores trabajaban la droga por gramos, para que otro tipo de suministradores le dieran salida. La distribución solía estar organizada a nivel de comunidades locales, siguiendo la organización tradicional de la subcultura criminal de Glasgow. En Edimburgo se conoce una estructura simila. Hasta mediados de los 80, la heroína llegaba a Edimburgo en dosis de una onza o más. Esto fue cambiando gradualmente debido a la distribución por parte de pequeños traficantes, que utilizaban a los camellos-consumidores. A finales de los 80, el mercado de Glasgow era mucho más complejo que el de Edimburgo, debido a las relaciones que se habían establecido con los suministradores al por mayor de Inglaterra. Las dos formas más frecuentes de costearse el consumo de heroína eran robar o actuar de camello y los pequeños hurtos, los cuales, se consideraban menos arriesgados que otras actividades delictivas más rentables.

A finales de los 80, la detención de numerosos camellos de heroína, los altos niveles de infección por HIV y un aumento importante de las prescripciones de sustancias sustitutivas dieron lugar a una disminución del suministro y del consumo de heroína en Edimburgo, lo cual reforzó la tendencia a un aumento del consumo de fármacos. La "farmacopea ilegal" ha cambiado en gran medida y está sujeta a continuos cambios (Hammersley et al., 1990). Antes de que se identificara el HIV, los escoceses se iniciaban en el consumo de heroína inyectándosela, o bien llegaban rápidamente a ese estadio. A partir de 1984, en Glasgow y en Edimburgo, el consumo por vía intravenosa de fármacos, como la buprenorfina, el temgesic, el temazepam y los tranquilizantes aumentó muy rápidamente y llegó a ser un grave problema en ambas ciudades (Haw, 1985; Haw y Liddell, 1987).

Desde 1987, el consumo de droga inyectable en Edimburgo ha disminuido notablemente, hasta el punto de ser la ciudad de Escocia con el índice más bajo de esta modalidad de consumo. (Scottish Drug Misuse Database 1955a)

La experiencia de Escocia pone de manifiesto que los preparados para el suministro por vía oral no necesariamente se utilizan de este modo. El comercio y el intercambio son muy frecuentes entre los toxicómanos. La metadona y la benzodiazepina que pueden obtenerse con receta médica no son siempre las substancias preferidas por el usuario, que puede intercambiarlas por otros productos. Los datos sobre la situación en Edimburgo señalan que, en los últimos años, la metadona, la dihidrocodeína, el temazepam, el diazepam, el triazolam y el temgesic han sido todos objeto de intercambio en el mercado negro. Hace quince años las sustancias opiáceas de mayor circulación en Edimburgo eran la heroína y el dipipanone. Mientras que ahora es más difícil encontrar esas drogas en la ciudad, se ha desarrollado un mercado negro ilegal de benzodiazepina. (El cannabis se usa como droga recreativa entre grupos de edad y clase social diversas, mientras que el LSD y el MDMA (éxtasis) se hicieron populares entre los jóvenes escoceses a principios de los 90, a raíz del fenómeno "rave"). En Glasgow el consumo de droga se ha extendido casi tanto como en Edimburgo. El temgesic y el temazepam se han hecho muy populares, al igual que la heroína, que es más fácil de encontrar en Glasgow y Aberdeen que en Edimburgo o Dundee.

\section{DESVIACIÓNY DISTRIBUCIÓN}

Después del cannabis, los fármacos son, al parecer, las drogas que más se utilizan en Escocia para usos no terapéuticos; en Inglaterra y en Gales, al cannabis le siguen las anfetaminas y las sustancias opiáceas adulteradas, y los fármacos ocupan el tercer puesto. A pesar de la creciente preocupación que despiertan en Inglaterra las drogas que pueden obtenerse con receta y adquirirse en las farmacias, parece que el problema es más grave en Escocia (Home Office, 1994). La conexión con el mercado ilegal de fármacos podría producirse mediante intercambios ilícitos de fármacos a nivel de ventas al por mayor, robos a fabricantes y farmacéuticos y adquisición directa en las far- 
macias. Casos judiciales recientes en Edimburgo y Londres han sacado a la luz conexiones delictivas en gran escala de ventas de productos adulterados y exportaciones de millones de pastillas.

Incluso durante un tratamiento, y durante largos períodos de tiempo, muchos pacientes siguen consumiendo drogas no prescritas, que obtienen de diversas fuentes con fines recreativos o para ir aumentando su propia dosis. Como cabría esperar, el precio de las drogas de importación ilegal suele ser más elevado en Escocia que en Londres, al ser esta ciudad el centro principal de venta y distribución de toda Gran Bretaña. La distancia geográfica y la reducción de los canales de suministro aumentan el coste del producto. Por la misma razón, en Edimburgo se alcanzan precios más altos que en Glasgow, ya que esta ciudad tiene un mercado de venta más amplio y una red de distribución más desarrollada. En Edimburgo, los distribuidores de heroína de nivel medio no se han recuperado plenamente de los efectos de una serie de detenciones realizadas a mediados de los 80 , de la propia drogodependencia ni de las consecuencias de los contagios de HIV por inyección que han sufrido algunos de ellos. Los más implicados en la criminalidad han optado por otro tipo de actividades ilegales.

\section{ESCOCIA, INGLATERRA Y GALES}

En general, el perfil del escocés típico sujeto a problemas físicos, psicológicos, sociales y legales asociados al consumo de drogas es el de un varón, de entre 20 y 30 años, desempleado, que vive en zonas urbanas pobres, que se inyecta varios de preparados ilegales y farmacéuticos y con un alto riesgo de infección por HIV. En muchos aspectos, la situación de Escocia es muy diferente de la de Inglaterra y Gales. En comparación con sus homólogos ingleses, los toxicómanos escoceses registrados oficialmente en el Ministerio del Interior británico por parte de médicos especialistas como adictos a un número limitado de sustancias opiáceas y a la cocaína son, probablemente, más jóvenes, inyectores y más expuestos a posibles denuncias. El volumen de contagio por HIV asociado al consumo de drogas es mucho más elevado en Escocia, lo que conlleva consecuencias inmediatas para los servicios legales y de otro tipo, así como para los propios toxicómanos.

Además, las modalidades de consumo en Escocia presentan diferencias sustanciales. Glasgow, Edimburgo, Dundee y Aberdeen son las ciudades con mayores problemas de drogas. En Glasgow se registran niveles muy altos de consumo de heroína, de consumo por intravenosa y de muerte por sobredosis (Scottish Drug Misuse Database, 1994; Home Office, 1994). En Escocia, en 1994, se produjeron 139 muer- tes de toxicómanos y supuestos toxicómanos por droga (Scottish Drug Misuse Databse, 1995a). Glasgow tiene una tasa de mortalidad por consumo de heroína particularmente elevada. Tanto en Glasgow como en Edimburgo se han propuesto estudios cooperativos sobre la mortalidad que contribuirían a esclarecer la forma en que la modalidad de consumo, el tipo de droga utilizado, las condiciones del HIV, el acceso a los servicios y las políticas de prescripción repercuten en la mortalidad asociada al uso de drogas en ambas ciudades.

\section{INFECCIÓN POR HIV}

La epidemiología relativa al HIV en Edimburgo y en la región de Lothian está cambiando del consumo de droga por intravenosa a la transmisión por contacto sexual. Desde diciembre de 1995, 1.105 residentes en Lothian han sido declarados seropositivos (Scottish Centre for Infection and Environmental Health, 1986). El registro de seropositivos de Lothian indica que, hasta octubre de 1995, 1.310 personas procedentes de Lothian, o de otras regiones, se han realizado las pruebas o han sido tratados contra el HIV en los servicios locales. Los focos de la epidemia siguen siendo determinados distritos de la ciudad de Edimburgo y, sobre todo, las zonas extremadamente pobres.

En los últimos tres años, se han producido en Lothian entre 50 y 75 nuevos casos de contagio de HIV al año (Scottish Centre for Infection and Environmental Health, 1996). En esta región, en la que vive solo el $14 \%$ de la población escocesa, se han registrado, en el período 1994-1995, el 44\% de los casos de SIDA (169) de Escocia (Lothian Health, 1995). Las parejas de los actuales o ex toxicómanos afectados por HIV son los de mayor riesgo, puesto que los toxicómanos de Edimburgo que contrajeron el HIV en los primeros años 80 han empeorado y son cada vez más contagiosos.

Afortunadamente, el nivel del consumo de drogas por intravenosa ha disminuido en gran medida, aunque continúa el uso de heroína y anfetaminas inyectadas. Por otra parte, en algunas zonas rurales se han identificado grupos de toxicómanos inyectores y, al parecer, en las cárceles escocesas existe un intercambio a gran escala de drogas y útiles en gran escala (McKenna 1993; Bird et al., 1995; Taylor et al., 1995)

\section{EL HIVY EL CONSUMO DE DROGAS POR INTRA- VENOSA}

De los 1.105 residentes seropositivos de Lothian, 596 (el 54\%) han contraido la infección inyectándose la 
droga (Scottish Centre for Infection and Environmental Health 1996). Sin embargo, los nuevos contagios de HIV asociados al uso de drogas han disminuido sustancialmente desde finales de los años 80 . En el período 1994-95, sólo 9 diagnosis de SIDA estaban relacionadas con el uso de drogas (Lothian Health, 1995). Las conclusiones de un estudio sobre la incidencia del HIV, realizado por el Consejo de Investigación Médica de Edimburgo, han puesto de manifiesto una tasa invariable de expansión del virus del 19\% (95\% límites de confianza del 15,7-22,9\%) en un período de tres años (1992-1994) entre toxicómanos activos (Richardson et al., 1995). Resulta alentador el hecho de que el 93\% de las personas que dieron positivo en pruebas anónimas estuvieran al corriente de su seropositividad. Más del $50 \%$ de las personas entrevistadas entre 1992 y 1994 se inyectaban menos de una onza a la semana y el $73 \%$ no había utilizado jeringuillas usadas en los seis meses anteriores (Richardson et al., 1995).

En los últimos quince años, se ha llevado a cabo en Escocia una investigación exhaustiva sobre las drogas, enmarcada en el ámbito universitario y en el de un servicio de suministro. Recientemente, un grupo de expertos oficiales ha fomentado otras encuestas a nivel local y nacional (Ministerial Drugs Task Force, 1994). Se han preparado estudios sobre la expansión de las drogas, con objeto de evaluar la magnitud del problema del consumo en Edimburgo y en Lothian. Sobre esta cuestión se habían realizado en Glasgow (1984), en Edimburgo (1986) y en Lothian (1993) investigaciones anteriores. El estudio más reciente, que ha contribuido a lo que de hecho ha dado lugar a un ciclo nacional, se realizó en Tayside en 1996 (Haw, 1985; Haw y Liddell, 1987; Haw, 1993; McKeganey, 1996). Hasta hace poco tiempo, el consumo de drogas por intravenosa constituía el objeto principal de las encuestas, por el hecho de estar asociado a la infección del HIV.

\section{EL HIVY LA POBLACIÓN CARCELARIA}

El consumo de droga inyectable en el ámbito de la población carcelaria es motivo de gran preocupación. El estudio sobre el aumento de inyectores seropositivos en Edimburgo ha revelado que 195 (el 31\%) de los toxicómanos por intravenosa había estado en prisión en los seis meses precedentes y que 35 (el 18\%) de ellos se habían inyectado durante el período de detención (Richardson et al., 1995). El 65\% (23) de los que se habían inyectado en la cárcel utilizaron para ello jeringuillas usadas y el $71 \%$ (25) se las habían intercambiado con otros presos. Durante el primer año del estudio, se ha puesto de manifiesto que los toxicómanos seropositivos que han estado más de una vez en la cárcel estaban 3,7 veces más expuestos al contagio que los que no habían estado (Davies et al., 1995). Desde 1993, en la cárcel de Saughton, en Edimburgo, se lleva a cabo un programa terapéutico de reducción del uso de drogas. El punto interesante es que la introducción de pruebas obligatorias en las cárceles británicas podría resultar, en muchos casos, una práctica adecuada en el marco de los programas de tratamiento en Escocia. El Servicio que funciona en las cárceles escocesas espera poder garantizar que dichos programas de tratamiento sean compatibles con los servicios existentes en la comunidad externa (Scottish Prison Service, 1996). A las instituciones sanitarias y sociales, así como al voluntariado, se les estimula a que participen en la implementación de los programas; asimismo, se ha establecido una conexión entre el servicio de asistecia en las cárceles y los de otras instituciones de tratamiento y prevención.

\section{EL HIVY LA TRANSMISIÓN HETEROSEXUAL}

La posibilidad de transmisión heterosexual a las parejas de los toxicómanos seropositivos sigue siendo elevada. Cincuenta y cuatro de los que constituyen el patrón de toxicómanos seropositivos del estudio sobre la extensión del HIV en Edimburgo nunca hace uso de preservativos con las parejas fijas y el $28 \%$ ni siquiera lo hace con las ocasionales (Davies et al., 1995). En conjunto, el 13,8\% (152/-1.105) de los contagios registrados en Edimburgo y en Lothian hasta finales de 1995 tuvo lugar por transmisión heterosexual (Scottish Centre for Infection and Environmental Health, 1996). Aunque en números absolutos no sea muy elevado, el contagio heterosexual ha contribuido en un $30 \%$ a los nuevos contagios en el período $1994-95$ y en un $24 \%$ en el período 1993-94 (Lothian Health, 1995; Lothian Health 1994). El Registro de las infecciones por HIV en Lothian indica que, en los últimos tres años, 34 personas han contraído el SIDA dentro de los 12 meses siguientes de haberse realizado la primera prueba de HIV. Diecisiete (50\%) de ellas se han contagiado por contacto heterosexual. Al parecer, los contagiados por contactos sexuales son menos conscientes del riesgo de transmisión y se someten a las pruebas únicamente cuando han desarrollado una enfermedad asociada al HIV. Por desgracia, es un hecho que las personas que están al corriente de su seropositividad deben adoptar prácticas seguras en el consumo de drogas y en los contactos sexuales.

\section{CASOS DE SIDA}

Hasta el 31 de diciembre de 1995, en Edimburgo y en Lothian se habían contabilizado 340 casos de SIDA, 
que representan el $46,5 \%$ de los registrados en toda Escocia (Scottish Centre for Infection and Environmental Health, 1996). Las categorías de riesgo reflejaban la evolución que seguía la epidemia del HIV en Edimburgo donde, entre 1982 y 1984, se contagiaron en primer lugar los gays, seguidos de un grupo de toxicómanos. Las encuestas demuestran que la expansión del SIDA debería ser más lenta entre los toxicómanos seropositivos que entre otros grupos de riesgo (Brettle et al., 1995), si bien, debido a la profilaxis, algunas personas infectadas por HIV han desarrollado una enfermedad sintomática e, incluso, han muerto sin haber desarrollado el SIDA propiamente dicho. Los toxicómanos seropositivos también han sufrido un alto índice de mortalidad debido a suicidios y actos violentos, así como a los efectos sinérgicos del HIV: sobredosis, pulmonías, hepatitis y septicemia.

\section{SERVICIOS DE DISTRIBUCIÓNY ACEPTACIÓN}

Hace diez años, se realizó en Escocia una débil tentativa de examinar la labor desarrollada por los servicios para la droga, la eficacia de las actuaciones y el impacto sobre las comunidades de toxicómanos. Ello puso de manifiesto una falta de interés de las autoridades con respecto a las drogas, así como la hostilidad de un buen número de psiquiatras, médicos y grupos comunitarios contra la prescripción de sustancias sustitutivas, antes de que se identificara en Edimburgo, en 1985, una epidemia de HIV asociada al uso de drogas. La aprobación de políticas dirigidas a la reducción de los daños, la institucionalización del intercambio de jeringuillas, la creación de un servicio especializado, la movilización de expertos médicos y sanitarios y el incremento del voluntariado, son todas medidas derivadas de la crisis del HIV. La situación del contagio por HIV en Edimburgo es más equiparable a la de España, Francia meridional e Italia que a la del resto de Gran Bretaña, donde la transmisión del HIV se produce sobre todo por contacto sexual (Lewis, 1992). En 1994, el contagio por HIV en Edimburgo estaba relacionado, en particular, con personas de entre 27 y 36 años que habían comenzado a inyectarse droga entre 1980 y 1987, y más concretamente entre 1982 y 1984, que la habían consumido durante más de siete años desde 1979, que habían compartido jeringuillas con otros toxicómanos seropositivos entre 1980 y 1987, que habían estado en prisión y utilizado jeringuillas de otros encarcelados y que residían en la zona norte de Edimburgo (Davis et al., 1995). Ahora se observa una creciente preocupación por los niveles de contagio de la hepatitis $C$ entre los toxicómanos y sus parejas. La tasa de contagio entre inyectores se estima en un 80\% (Brettle 1995).

La estrategia para combatir la droga ha seguido el modelo de reducción de los daños. Los objetivos prin- cipales eran reducir la incidencia de los contagios asociados al uso de drogas, reducir los daños físicos, psicológicos, legales y sociales relacionados con dicho uso y estimular a los toxicómanos a practicar la abstinencia. El Consejo Asesor sobre Abuso de Drogas (ACMD) de Gran Bretaña sostiene que "los bajos niveles de comportamientos de riesgo y la baja incidencia del HIV entre los toxicómanos proporcionan una base lo suficientemente sólida como para poder afirmar que la estrategia adoptada en Gran Bretaña ha resultado un éxito y que esos niveles contrastan claramente con los que se han observado en otros países europeos" (ACMD, 1993, p.2). Desgraciadamente, la aplicación de dichas medidas ha llegado demasiado tarde para los toxicómanos seropositivos de Edimburgo que ya han contraído el SIDA, así como para sus parejas.

En la región de Lothian, la componente epidemiológica del HIV relacionada con el uso de drogas es tan alta, que los toxicómanos o ex toxicómanos reciben atención tanto de los servicios relacionados con el HIV como de los relacionados con la droga. La iniciativa contra el intercambio de jeringuillas que lleva a cabo el Equipo para la Reducción de los Daños está dirigida, en particular, a los inyectores. El Equipo, además de realizar una labor de carácter preventivo entre los toxicómanos seropositivos y los varones homosexuales, colabora con el Servicio Comunitario para los Problemas de la Droga, el cual presta asistencia a todos los toxicómanos, a pesar de que ambas instituciones son bastante diferentes.

El Informe de la Base de Datos de Escocia para el Abuso de Drogas, de 1994, señala que, en la región de Lothian, la edad de los toxicómanos (940) que buscan ayuda para resolver sus problemas es similar a la del resto de Escocia. El $60 \%$ de los pacientes de los servicios tiene entre 20 y 29 años. El 64\% son varones y el $82 \%$ parados. En Lothian, sólo el $5 \%$ de la muestra optó por la heroína la primera vez que consumió droga. Lo que diferencia a Lothian de otras zonas es que el consumo de preparados por vía oral es superior al consumo de drogas inyectables. El estudio sobre la magnitud del problema de la adicción y las prácticas generales de consumo en el período 199394 (Scottish Drug Misuse Database, 1995b) ha puesto de manifiesto una tendencia a evitar el uso por intravenosa. Sin embargo, un grupo importante de inyectores sigue intercambiándose jeringuillas, por lo que son necesarias nuevas iniciativas para llegar a este grupo de toxicómanos de riesgo.

\section{SERVICIOS COMUNITARIOS PARA PROBLEMAS RELACIONADOS CON LA DROGA}

El Servicio Comunitario para los Problemas de la Droga (CDPS), que presta asistencia en colaboración con los médicos internistas, se creó en 1988. Los pro- 
gramas de asistencia están diseñados para reducir los daños que el abuso de drogas produce en el toxicómano, en su familia y en la comunidad. La drogadicción se considera una condición recidiva, que requiere una amplia gama de actuaciones, desde la prevención a la intervención precoz, desde la reducción de los daños hasta el tratamiento terapéutico. Este Servicio ha utilizado en gran medida la prescripción de sustancias sustitutivas a largo plazo, así como programas flexibles de estabilización, adaptados a las necesidades individuales. La inyección segura, la eliminación de la inyección, el uso seguro de drogas por vía oral, el consumo reducido y la abstinencia se consideran todas ellas fases de objetivos importantes. Los toxicómanos reciben asistencia de un gran número de estructuras comunitarias, entre las que cabe mencionar los centros sanitarios, los departamentos sociales e, incluso, el ámbito de su propio domicilio. A los adictos al opio y/o a la benzodiazepina se les facilita generalmente preparados terapéuticos sustitutivos, con objeto de disuadirles de que sigan haciendo un uso ilegal de las drogas, en particular, por intravenosa. El médico internista del toxicómano (GP) es quien suele recetar los fármacos, siguiendo las recomendaciones del servicio de atención especializado. Los programas de tratamiento están sujetos a revisiones periódicas, tras una descripción detallada del grado de adicción.

Se han preparado informes clínicos y cuestionarios para el alta médica, dando información sobre el comportamiento del paciente, sus hábitos de adición y otros detalles. A los internistas se les insta a que prescriban metadona por vía oral y otras sustancias sustitutivas, con objeto de reducir el consumo por intravenosa y la expansión del HIV. Más del 70\% de los médicos de Edimburgo han expedido recetas a cerca de 1.600 toxicómanos que desde 1988 han cambiado su modalidad de consumo a favor de los fármacos por vía oral. Las tasas de infección por HIV entre los que han acudido al Centro han ido disminuyendo paulatinamente. (Community Drug Problem Service, 1994; Bury, 1994; Bury, 1996; Haw, 1993).

Los controles internos y las evaluaciones que se han llevado a cabo ponen de manifiesto los óptimos resultados que se han conseguido en lo que se refiere al consumo por intravenosa, a la criminalidad, a la prostitución y a los niveles de consumo de opiáceos. El $31 \%$ de un grupo (256) que el CDPS evaluó en 1990 y 1991 ha seguido un tratamiento durante 12 meses como mínimo. Durante el tratamiento, no se han registrado infecciones por HIV en el ámbito de ese grupo.

Al 28\% de los pacientes se les ha dado el alta en condiciones satisfactorias y el $40 \%$ de los toxicómanos han seguido el tratamiento al menos durante seis meses (Community Drug Problem Service, 1994; Peters et al., 1994). Para fomentar sucesivos cambios de comportamiento en un grupo de toxicómanos y en una población más amplia, que apenas si usa preservativos, es necesario aplicar nuevos métodos de intervención. En la región de Lothian, las políticas relativas a la prescripción de fármacos precisan de revisiones periódicas, para mejorar una operación que ha demostrado ser de gran interés para los pacientes, los internistas y los órganos directivos.

\section{REDUCCIÓN DEL CONSUMO DE DROGAS POR INTRAVENOSA E INTERCAMBIO DE JERINGUI- LLAS}

Aunque las características sociodemográficas de las personas objeto de estudio por parte del Servicio Comunitario para los Problemas de la Droga (CDPS) se hayan mantenido invariables en el curso de los años, las modalidades de consumo en el período en estudio han ido cambiando paulatinamente, en particular durante los primeros cinco años de actividad del centro. El porcentaje de pacientes adictos que practicaban el intercambio de jeringuillas ha disminuido del $85 \%$ en 1988 , al $51 \%$ en 1993 . En el mes anterior (en el mismo período de tiempo) el intercambio ha bajado del 39 al $13 \%$.

La proporción de nuevos pacientes inyectores ha disminuido del $97 \%$ en 1988 , al $43 \%$ en 1993 . En el més anterior al estudio (en los mismos años) el consumo de droga inyectable entre los inyectores había bajado del $88 \%$ al $14 \%$. El estudio de los pacientes del CDPS en tratamiento durante seis meses $(n=74)$ y 24 meses $(n=57)$ ha demostrado que la tasa de consumo por intravenosa ha seguido disminuyendo (Greenwood, 1996).

\section{EL CDPS Y LAS TASAS DE SEROPOSITIVIDAD POR HIV}

La tasa de seropositividad de los pacientes examinados antes del estudio ha bajado del $21 \%$ en 1988 , al $8 \%$ en 1993. En el mismo período de tiempo, el uso de presentativos se ha mantenido invariable, lo que demuestra que no ha habido una tendencia equivalente hacia un comportamiento sexual seguro, a pesar de los importantes programas locales de educación sanitaria. El personal pone especial atención en fomentar la reducción del consumo por intravenosa y el intercambio de jeringuillas; sin embargo, evitar que el HIV u otros virus se propaguen por contacto sexual resulta particularmente difícil. El personal ha señalado que la reducción de los daños a nivel sexual es un argumento difícil de tratar con los pacientes. Es posible que los especialistas en el problema de la droga encuentren la misma dificultad al tratar el tema del sexo que el que 
encontrarían los especialistas en el aparato genital al tratar problemas relativos a la droga en Gran Bretaña y, probablemente, en otros lugares. Los medios de comunicación europeos señalan que es más difícil intentar cambiar el comportamiento sexual que el comportamiento en relación a las drogas. Los toxicómanos inyectores no conceden mayor importancia al sexo seguro de la que muchos atribuyen al consumo seguro de drogas. En este sentido, cabría señalar que al ser el sexo un elemento más importante para el comportamiento humano, también es más difícil modificar los comportamientos sexuales.

Gracias al apoyo especializado de los médicos internistas que ofrece el Centro, éste ha podido atender a un mayor número de pacientes del que hubiera sido posible con un servicio de prescripción centralizado. Si bien la mayor parte de los pacientes siguen consumiendo droga, ahora hacen uso de fármacos orales más seguros, con un período de caducidad mayor, y el $50 \%$ está siguiendo programas de reducción gradual. Actualmente, la mayor parte de los pacientes raramente se inyecta droga, con lo que se reduce el riesgo de contraer SIDA u otra infección relacionada con el consumo por vía intravenosa (Greenwood, 1996).

La tasa de transmisión del HIV entre los inyectores ha descendido, así como la del consumo por intravenosa y, en la actualidad, muchos toxicómanos hacen uso permanente de drogas por vía oral. Parece que en la región de Lothian hay un gran porcentaje de adictos que está en contacto con los servicios de asistencia. El consumo de heroína está en baja y, aunque podrían señalarse diversas razones para este mejora, es evidente que el régimen de prescripción, en el que han colaborado el CDPS y los médicos internistas, ha sido fundamental (Williamson, 1994).

\section{EL EQUIPO DE REDUCCIÓN DE DAÑOS}

El Equipo de Reducción de Daños se creó en 1987 con el fin de proporcionar un servicio de intercambio de jeringuillas y de apoyo a las personas con riesgo de infección por HIV. El Equipo presta una serie de servicios, entre los que se incluyen el suministro de útiles para inyectarse y locales para practicar el consumo de drogas y el sexo seguros (con unidades móviles o fijas). La actividad actual del servicio comprende servicios directos o indirectos para el fomento del consumo de drogas y sexo seguros.

En el período 1994-95, este servicio atendió a 825 pacientes. Se registraron 2.287 contactos, 730 de los cuales $(31,5 \%)$ estaban relacionados con el intercambio de útiles para inyectarse, con actuaciones dirigidas al consumo seguro de drogas, al suministro seguro por intravenosa, a una eliminación más segura, al vendado de úlceras y abscesos, al intercambio de jerin- guillas y a la distribución de lejía. Recientemente, el Equipo ha adoptado la labor de facilitar/coordinar servicios generales y se ha centrado en actividades de especialización/innovación. Veinticinco farmacias de Lothian participan en el programa de intercambio de jeringuillas, facilitando a los toxicómanos material gratuito para inyectarse (Gavin, 1995). Los resultados del CDPS y de otros servicios de prescripción han demostrado que la demanda de una estrategia de intercambio de jeringuillas ha sido inferior en comparación con otras ciudades escocesas, por ejemplo Glasgow. La gran popularidad que esta estrategia ha alcanzado en Glasgow pone en evidencia que quizá esa erala única asistencia de tipo sanitario de que disponían los toxicómanos de esa ciudad en el pasado. Ahora se ha creado un Servicio para los Problemas con la Droga, con la colaboración de médicos internistas, patrocinado por la Oficina Escocesa, y conectado con Edimburgo.

\section{OTROS SERVICIOS PARA LOS TOXICÓMANOS}

Durante el período 1993-94, 1.119 personas se han dirigido a los servicios comunitarios de la región de Lothian, ofreciendo 9.475 interacciones con los pacientes (Centre for HIVIAIDS and Drug Studies, 1994). Los médicos internistas, el Departamento Social y el CDPS han sido los servicios más contactados por este orden, lo cual pone en evidencia el papel destacado que desempeñan los internistas en relación con los problemas de la droga. Las sustancias opiáceas y las benzodiacepinas son las que han ocasionado mayores problemas. Aunque también está presente el cannabis, el consumo que hacen los pacientes no parece que sea en sí mismo motivo de preocupación.

Las cuestiones que han afectado en mayor medida a la vida de los pacientes son, en primer lugar, las relativas a la droga y, en segundo lugar, las relaciones personales. Un dato importante es que la posibilidad de infección por HIV y sus consecuencias preocupaban a los pacientes menos que el dinero, la vivienda y los problemas legales (Centre for HIV/AIDS and Drug Studies, 1994). Este hecho pone en tela de juicio la prevención del HIV y la labor educativa. Puesto que los toxicómanos y las personas afectadas por HIV viven principalmente en zonas de extrema pobreza, muchos de ellos se preocupan por más las cuestiones prácticas de supervivencia cotidiana que por las posibles consecuencias del contagio por HIV.

\section{ESTUDIOY EVALUACIÓN}

Los servicios de la región para el HIV/SIDA y las drogas participan en un programa de estudio y evalua- 
ción en colaboración con los responsables del servicio y los compradores. El personal de investigación del Centro de Estudios sobre el HIV/SIDA y las Drogas, una unidad dentro del departamento médico de salud pública de la región de Lothian, realiza las evaluaciones externas. Los investigadores, y un número cada vez mayor de suministradores, reconocen que una autoevaluación eficiente, con las respuestas adecuadas unida a una evaluación externa objetiva, pueden fortalecer y reafirmar la labor del servicio. Otras cuestiones específicas se revisan a la luz de la evaluación y en colaboración con las instituciones suministradoras y de planificación.

En Edimburgo y en Lothian funcionan actualmente 12 servicios que actúan en la calle y con los residentes, así como en la prevención y asistencia de los problemas con las drogas. Cada uno de los servicios elabora una evaluación externa de hasta tres años. Inicialmente, las evaluaciones se centraban en el procedimiento, pero una vez que los servicios se han normalizado, son cada vez más frecuentes las medidas encaminadas a la obtención de resultados. Entre los "elementos de eficacia" se incluyen pacientes que se han desintoxicado, otros que se han estabilizado o que han reducido el consumo de droga, que han pasado de inyectarse droga a consumirla por vía oral, que han abandonado el intercambio de jeringuillas, que han mejorado su condición social y sus relaciones interpersonales, y otros que han reducido el número de dosis y de detenciones, así como las ocasiones en que se administran una sobredosis.

Los servicios clínicos que facilitan prescripciones para estabilizar o reducir el consumo de drogas están incidiendo en el comportamiento de riesgo asociado a la droga, así como en el estilo de vida. Los resultados que han logrado esos servicios son difíciles de evaluar. La flexibilidad con que el Equipo de Reducción de los Daños conduce los programas dirigidos a toxicómanos seropositivos difíciles de tratar y a varones que tienen relaciones homosexuales en lugares públicos ha demostrado su eficacia al conseguir alcanzar a grupos tan diferentes.

Las organizaciones de voluntariado autoevaluan anualmente su propia labor, sobre la base de las directivas establecidas conjuntamente. En las especificaciones detalladas sobre el servicio, exponiendo la necesidad de las evaluaciones y el ciclo de las mismas, se describen el tipo, el nivel y la calidad del servicio solicitado, así como los objetivos de las áreas específicas de actividad. Los servicios médicos, el Servicio para los Problemas con las Drogas y un centro de acogida durante las crisis de abstinencia, han establecido medidas adecuadas para alcanzar dichos resultados. Ello conlleva una mayor dificultad para las instituciones comunitarias debido, en parte, a que reduce su labor preventiva y, en parte, a la naturaleza de su actividad. Los resultados de las actuaciones a nivel territorial pueden ser especialmente difíciles de cuantificar, a pesar de que los programas educativos que se promueven entre las redes subculturales de toxicómanos tengan un efecto beneficioso considerable. Debido a esos límites, los datos cualitativos se consideran tan importantes como los cuantitativos.

\section{CONCLUSIÓN}

Los datos epidemiológicos ponen de manifiesto que las políticas de reducción de los daños, adoptadas en 1986, han limitado la expansión del HIV en la región de Lothian, así como las consecuencias del consumo de drogas por intravenosa (Scottish Centre for Infection and Environmental Health, 1996). Gracias a dichas evaluaciones, las instituciones han podido establecer objetivos más concretos para sus propios servicios. Es de esperar que la atención creciente que reciben las llamadas "medidas eficaces" garantice que cada individuo o comunidad pueda beneficiarse de la asistencia que se pone a su disposición y, a ese respecto, podría ser necesario normalizar las políticas y las directivas. También podría suceder que el bajo consumo de drogas entre la gente joven, que se inicia con fines recreativos pero que está muy extendido, se convierta con el tiempo en una actitud de desafío que vaya aumentando de forma gradual. Muchos jóvenes de Gran Bretaña no consideran que el cannabis, el éxtasis y las anfetaminas sean un problema. Sin embargo, la ilegalidad de esas sustancias crea dificultades a los consumidores y a la hora de aplicar la ley. Aunque en el ámbito de la asistencia, la prevención y la legislación se escuchan voces dispuestas a hacer frente a esos problemas, la falta de voluntad política de los legisladores no facilita el entorno adecuado para el debate. Existe la preocupación de que un reducido porcentaje de usuarios de drogas recreativas pueda involucrarse involuntariamente en consumos de droga más problemáticos o en conductas de alto riesgo. El debate sobre si la represión es la solución adecuada o no queda abierto.

La transmisión a través del uso de material para inyectarse contaminado es en la actualidad responsable de un pequeño porcentaje de nuevos contagios por HIV. La transmisión heterosexual por parte de toxicómanos seropositivos constituye un gran reto para la atención y los servicios sanitarios competentes. En este momento, existe un preocupación creciente respecto a los altos niveles de infección de hepatitis $C$ entre la comunidad de toxicómanos seropositivos. Esto constituye un reto mayor para la asistencia médica, así como una necesidad de estrategias claras y de protocolos concretos. 


\section{BIBLIOGRAFÍA}

Advisory Council on the Misuse of Drugs (1993) AIDS and Drug Misuse Update. London: Her Majesty's Stationery Office.

Barnard, M., and N. McKeganey (1990) Adolescents, sex and injecting drug use: risks for HIV infection. AIDS Care. 2 (2):103-116.

Bird, A.G., S.M. Gore, S. Cameron, A.J. Ross and D.J. Goldberg (1995) Anonymous HIV surveillance with risk factor elicitation at Scotland's largest prison, Barlinnie. AIDS. 9, (7):801-803.

Bird, A.6, S.M. Gore, D.W. Jollify, S.M. Burns (1992) Anonymous HIV surveillance in Saughton Prison, Edinburgh. AIDS 6(9): 725-733.

Brettle, R.P. (1995) Hepatitis C - policy considerations. Centre for HIVIAIDS and Drug Studies. Policy Seminar, City Hospital, Edinburgh, March 23.

Brettle, R.P., A.J. McNeil, S.M., Gore, A.G. Bird, C.L. Leen, and A. Richardson (1995) The Edinburgh City hospital cohort: analysis of enrolment, progression and mortality by baseline. Quarterly Journal of Medicine 88:479491.

Bury, J. (1994) HIV Infection and drug misuse in Lothian general practice, report on epidemiology questionnaire 1993. Edinburgh: Lothian Health.

Bury, J. (1996) HIV Infection and drug misuse in Lothian general practice, report on epidemiology survey 1995. Edinburgh: Lothian Health Primary Care Services.

Centre for HIV/AIDS and Drug Studies (1994) Data analysis of voluntary agency evaluation sheets. Edinburgh: Lothian Health Board.

Community Drug Problem Service (1994) Lothian 's Community Drug Problem Service, a five year review 19881993. Edinburgh: Edinburgh Healthcare Trust.

Davies, A.G., N.J. Doomy, A. Peters, G.E. Bath, S.M. Burns, and A.M. Richardson (1995) HIV in injecting drug users in Edinburgh: prevalence and correlates. Journal of Acquired Immune Deficiency Syndrome and Human Retro-virology. 8, 399-405.

Gavin, S. (1995) An evaluation of the Harm Reduction Team. Edinburgh: Centre for HIV/AIDS and Drug Studies.

Greenwood, J. (1996) Six years' experience of sharing the care of Edinburgh's drug users. Psychiatric Bulletin. 20, 8-11.

Hammersley, R., T. Lavelle, and K.A. Forsyth (1990) Buprenorphine and temazepam abuse. British Journal of Addiction; 84:301-303.

Haw, S. (1985) Drug problems in Greater Glasgow. London: Standing Conference on Drug Abuse.

Haw, S. (1993) Pharmaceutical drugs and illicit drug use in Lothian region. Edinburgh: Centre for HIVIAIDS and Drug Studies.

Haw, S., and D. Liddell (1987) Drug problems in Edinburgh District. London: Standing Conference on Drug Abuse.
Home Office (1994) Area Tables. Statistics of the Misuse of Drugs: Addicts notified to the Home Office UK 1993. London: Home Office.

Lewis, R. (1992) Misuse of drugs in Scotland. Proceedings of the Royal College of Physicians. Edinburgh 22: 1828.

Lothian Health (1994) HIV/AIDS in Lothian - Strategies for survival. AIDS (Control) Act report for 1993/94. Edinburgh: Lothian Health Board.

Lothian Health (1995) H1V/AIDS in Lothian -Ten years on. AIDS (Control) Act report for - 1994/95. Edinburgh; Lothian Health Board.

McKeganey, N. (1996) Director, Centre for Drug Misuse Research, University of Glasgow, personal communication.

McKenna, C. (1993) Problem drug use and related needs in East Lothian. Glasgow: Scottish Drugs Forum.

Ministerial Drugs Task Force (1994) Drugs in Scotland: Meeting the Challenge. Edinburgh: Scottish Office Home and Health Department.

Peters, A.D., M.M. Reid, S.G. Griffin (1994) Edinburgh drug users: are they injecting and sharing less? AIDS 8(4): 521-528.

Richardson, A.M., A.G. Davies, A. Peters, and N. Dominy (1995) Serial prevalence study of HIV infection and HIV risk behaviour among injecting drug users in Edinburgh 1992-94. Answer AM-17 WR 95/47.

Robertson, J.R., and A.B. Bucknall (1986) Heroin users in a Scottish city. Final report for Scottish Office Home and Health Department. Edinburgh: Scottish Home and Health Department.

Scottish Centre for Infection and Environmental Health (1996) HIV infection and AIDS: Quarterly report to 31st December 1995. Answer AM-19 96/04.

Scottish Drug Misuse Database (1994) Bulletin. Edinburgh: Information Services Division, Scottish Health Service Common Services Agency.

Scottish Drug Misuse Database.

1995a Bulletin. Edinburgh: Information Services Division, Scottish Health Service Common Services Agency.

Scottish Drug Misuse Database (1995b) Monitoring of problem drug use seen in general practice. Edinburgh: Information Services Division, Scottish Health Service Common Services Agency.

Stewart, D. (1996) Presentation on Mandatory Drug Testing. Scottish Prisons Service Seminar. Saughton Prison, Edinburgh. 23rd February.

Taylor, A., D. Goldberg, J. Emslie, J. Wrench, L. Gruer, S. Cameron, J. Black, B.

Davis, J. Macgregor, E. Follett, J. Harvey, J. Basson, and J. McGavigan (1995) Outbreak of HIV infection in a Scottish prison. British Medical Journal 310:289-292.

Williamson, L. (1994) An evaluation of the Community Drug Problem Service. Edinburgh: Centre for HJV/AIDS and Drug Studies. 
